\title{
How Search Engines Handle Suicide Queries
}

\author{
Olivia Borge, Victoria Cosgrove, Elena Cryst, Shelby \\ Grossman, Shelby Perkins, and Anna Van Meter
}

\begin{abstract}
The suicide contagion effect posits that exposure to suiciderelated content increases the likelihood of an individual engaging in suicidal behavior. Internet suicide-related queries correlate with suicide prevalence. However, suicide-related searches also lead people to access help resources. This article systematically evaluates the results returned from both general suicide terms and terms related to specific suicide means across three popular search engines-Google, Bing, DuckDuckGoin both English and Spanish. We find that Bing and DuckDuckGo surface harmful content more often than Google. We assess whether search engines show suicide prevention hotline information, and find that 53\% of English queries have this information, compared to $13 \%$ of Spanish queries. Looking across platforms, 55\% of Google queries include hotline information, compared to $35 \%$ for Bing and $10 \%$ for DuckDuckGo. Specific suicide means queries are $20 \%$ more likely to surface harmful results on Bing and DuckDuckGo compared to general suicide term queries, with no difference on Google.
\end{abstract}

\section{Introduction}

The suicide contagion effect posits that exposure to suicide-related content increases the likelihood of an individual engaging in suicidal behavior; this is especially true when the deceased is a relatable person (i.e., a celebrity or peer) (Joiner Jr 1999; Randall, Nickel, and Colman 2015). The contagion effect is supported by a number of studies, including research on the Netflix series 13 Reasons Why. The series, about a teenage girl who dies by suicide and the ways she seeks revenge on the people whose behavior is implicated in her death, has been criticized for (among other things) its graphic presentation of the suicide, which is strongly discouraged by suicide prevention groups (Reporting on Suicide, n.d.). Suicide rates among young people increased after the show's release (Bridge et al. 2020; Niederkrotenthaler et al. 2019; Sinyor et al. 2019). Suicide queries on Google also rose following the series premiere (Ayers et al. 2017). Internet suicide-related queries correlate with suicide prevalence (Arendt, Scherr, and Romer 2019). However, suicide-related searches also lead people to access help resources when presented (Mars et al. 2015). This suggests that the prioritization of search results has important implications for individual suicide risk. A recent study assessed the consistency of support messaging at the top of suicide-related search results on Google; messages 
were shown less than one-third of the time for harmful suicide-related searches (Arendt, Haim, and Scherr 2020).

The goal of this article is to systematically evaluate the results returned from both general suicide terms and terms related to specific suicide means across three popular search engines-Google, Bing, DuckDuckGo-in both English and Spanish. We compare the prioritization of pro-suicide results (i.e., the presence of content encouraging suicide or discussing suicide options in the top 20 search results); the presence of suicide prevention content (e.g., suicide prevention hotline information), both from the search engine and within websites; ads related to suicide; and recommended searches. Understanding how well search platforms identify and address suicide-related searches can inform efforts to reduce the harmful effects of online content with significant public health implications.

We find that Bing and DuckDuckGo surface harmful content more often than Google. Results for English queries, surprisingly, are more harmful than results for Spanish queries on Bing and DuckDuckGo, with no difference on Google. However, we also assess whether the search platforms show suicide prevention hotline information, and find that on average $53 \%$ of English queries have this information, compared to $13 \%$ of Spanish queries. Looking across platforms, $55 \%$ of Google queries include hotline information, compared to 35\% for Bing and 10\% for DuckDuckGo. Specific suicide means queries are $20 \%$ more likely to surface harmful results on Bing and DuckDuckGo compared to general suicide term queries, with no difference on Google. Looking only at the harmful URLs, we find that $45 \%$ of these URLs are blogs. We collect data on advertisements shown for each query, and find no harmful advertisements in the dataset. Last, we look at recommended searches, and find that $44 \%$ of Bing's recommended searches for English queries relate to encouraging self-harm or harm to animals.

\section{Self-Harm and the Internet}

Online activity, including social media, has been highlighted as a possible risk factor for suicide, especially among young people (Luby and Kertz 2019; Massing-Schaffer and Nesi 2020). The suicide contagion effect is well documented (Velting and Gould 1997; Daine et al. 2013; Joiner Jr 1999); exposure to suicide-related information is associated with increased suicide prevalence, including both fictional (Sugg et al. 2019; Niederkrotenthaler et al. 2019; Sinyor et al. 2019; Ayers et al. 2017) and real (Joiner Jr 1999; Won et al. 2013; Sisask and Värnik 2012) accounts of suicide deaths. Individuals who specifically seek self-harm or suicide-related content may be at especially high risk for this contagion (Sedgwick et al. 2019; Mars et al. 2015; Daine et al. 2013). Although some online platforms (e.g., Facebook [2021] and TikTok [n.d.]) forbid self-harm-related content (Eggertson 2015), these rules are limited in effect (Sumner, Burke, and Kooti 2020; Carlyle et al. 2018; Khasawneh et al. 2020), and coexisting chat rooms and discussion boards provide a space to discuss suicide openly, including to provide instructions for how to make a fatal attempt (Mars et al. 2015; Becker and Schmidt 2004). Visitors to these sites are at elevated risk for suicide (Arendt, Scherr, and Romer 2019; Dunlop, More, and Romer 2011; Marchant et al. 2017).

Individuals are likely to find suicide chat rooms and other pro-suicide websites through search platforms. People with mental health concerns (Van Meter et al. 2019; Birnbaum et al. 2017), including those who go on to engage in suicidal behavior, seek information online (Moon et al., in press). What they find can directly influence their behavior; changes in the frequency of suicide-related searches have been linked to increased deaths at the population level (Arendt and Scherr 2017). However-perhaps counterintuitively-people 
who go online to search suicide-related topics report being more likely to access sites offering help-if they are available (Mars et al. 2015). This suggests that prioritizing search results that highlight support services could reduce online suicide contagion.

Due to its market monopolization, Google has been singled out as a "gatekeeper" with a responsibility to protect people from suicide contagion online (Arendt, Haim, and Scherr 2020; Kirtley and O'Connor 2020). In response, Google implemented a "Suicide Prevention Result" (SPR) initiative. SPR, which began in 2010, promises to post the National Suicide Prevention Hotline at the top of the results for searches deemed suicide-related. Taken at face value, policies like SPR are well intentioned. However, the effectiveness of online suicide prevention efforts (e.g., mental health awareness messages and online support groups) has not been established (Christensen, Batterham, and O'Dea 2014; Lai et al. 2014; Klimes-Dougan, Wright, and Klingbeil 2016), while the harmful effects of suicide-related content are well known. Based on decades of evidence, removing or demoting search results with problematic content would likely be more effective than posting suicide hotline information.

There are ethical concerns related to companies implementing suicide prevention policies given the fact that little is known about how they work (Arendt and Scherr 2017). Furthermore, it is unclear how consistently these policies are applied; Google searches with less explicit suicide content (e.g., about celebrity suicides) and those conducted in languages other than English tend to be missed by the algorithm (Arendt, Haim, and Scherr 2020; Kirtley and O'Connor 2020). Searches may also be conducted through other platforms. For example, DuckDuckGo, which promotes itself as a more private option for searching, and Bing, which is integrated with Microsoft products, are also widely used. Existing research suggests that the Bing search engine does less content moderation than Google's search engine in many domains, including misinformation ("Bing's Top Search Results Contain an Alarming Amount of Disinformation" 2019) and child exploitation (Constine 2019; Keller and Dance 2019). Additionally, Bing and DuckDuckGo, unlike Google, have no public self-harm policies (Perkins, Cryst, and Grossman 2021).

Given the vast range of search terms that could be used in a suicide-related search, it is unlikely that steps taken to mitigate the exposure to harmful search results are equally effective across searches. Algorithms designed to identify terms like "suicide" or "self harm" may miss searches about means, which could pose a greater risk to individual safety (Moon et al., in press). In fact, if searches not only fail to return a suicide prevention message (e.g., posting information about the National Suicide Prevention Hotline at the top of the results), but also return results that include suicide chat rooms or other potentially pro-suicide content, search results can elevate an individual's risk for suicide.

If search engine suicide prevention algorithms fail to identify English language searches related to means and/or searches that don't include obvious suicide terms, it is unlikely that searches performed in other languages are well categorized. Within the United States, Spanish is the second-most spoken language; comparing searches in Spanish and English conducted within the US can provide an estimate of how the risk for exposure to suicide content may vary by language.

\section{Data and Methods}

To create the dataset, we first identified 10 search queries. We included a mix of queries related to suicide that were popular according to Google Trends and SEMRush, a search engine optimization company (e.g., "suicide" and "kill yourself") and queries that re- 
search by Moon et al. (in press) has identified as being used by individuals who were later hospitalized for suicide thoughts and behaviors (e.g., "how painful is slitting your wrists" and "everything seems overwhelming and pointless"). We made sure to include queries related to the most commonly used strategy in suicide attempts, overdose/poisoning, and queries related to a more lethal strategy, suffocation. We also made sure our list included general and specific queries.

We ran the searches in March 2021 in English and Spanish, the two most commonly spoken languages in the US. Table 1 shows the list of queries.

\begin{tabular}{|l|l|l|}
\hline Query in English & Query in Spanish & $\begin{array}{l}\text { General / Specific / } \\
\text { Other }\end{array}$ \\
\hline $\begin{array}{l}\text { how painful is slitting your } \\
\text { wrists }\end{array}$ & $\begin{array}{l}\text { que tan doloroso es } \\
\text { cortarse las venas }\end{array}$ & Specific \\
\hline best pills to overdose on & $\begin{array}{l}\text { mejores pastillas para un } \\
\text { sobredosis }\end{array}$ & Specific \\
\hline $\begin{array}{l}\text { everything seems } \\
\text { overwhelming and } \\
\text { pointless }\end{array}$ & me quedé sin esperanzas & Other \\
\hline kill yourself & matarse & General \\
\hline suicide & suicidio & General \\
\hline hanging painful & es doloroso ahorcarse & Specific \\
\hline $\begin{array}{l}\text { where's the best place to } \\
\text { shoot yourself in the head }\end{array}$ & $\begin{array}{l}\text { donde está el mejor lugar } \\
\text { para dispararse en la } \\
\text { cabeza }\end{array}$ & Specific \\
\hline $\begin{array}{l}\text { easiest way to suffocate } \\
\text { yourself }\end{array}$ & $\begin{array}{l}\text { la manera más fácil de } \\
\text { asfixiarse }\end{array}$ & Specific \\
\hline suicide chatrooms & sala de chat de suicidio & General \\
\hline $\begin{array}{l}\text { what is the easiest way to } \\
\text { poison myself? }\end{array}$ & $\begin{array}{l}\text { cual es el modo más fácil } \\
\text { de enevenenarme }\end{array}$ & Specific \\
\hline
\end{tabular}

Table 1: Queries in English and Spanish, and whether the query was specific, general, or something else.

When running the Spanish queries, we wanted to imitate the environment of someone in the US who prefers to run searches in Spanish. Research suggests that search engines are not effective at identifying the language of queries and showing results in that same language ("Multilingual SEO: It's Actually a Pretty Big Challenge for Google to Determine the Language of a Query" 2014). We spoke to native Spanish speakers in the US who reported that they changed their language settings on search platforms. As such, for Spanish queries, we changed the language setting on the search platforms to Spanish. ${ }^{1}$

In translating the English search terms into Spanish, we aimed to keep the searches as comparable as possible. Differences in the structure of Spanish and common usage meant that some terms were not easily translated literally from English to Spanish, and we recognize that translation is inherently subjective. For example, the term "matarse"

1. For Google, we chose "español (Latinoamérica)," for Bing we chose "español," and for DuckDuckGo we chose "español (México)." 
in Spanish means both "kill yourself" and "kill oneself." Our translation that deviated most from the English query was the translation for "everything seems overwhelming and pointless." The literal translation of this phrase would be "todo parece agobiante y sin sentido." This phrase felt cumbersome and unnatural to the native speakers we consulted, and less likely to be typed into an online search. Alternate translation options included "la vida ya no tiene sentido" ("life doesn't have meaning"), "ya no puedo más" ("I can't anymore”), "ya no tengo fuerzas para seguir" ("I don’t have the energy to go on"), and "me quedé sin esperanzas" ("I am left without hope"). We chose the latter as it most approximated the sentiment of the original phrase. We recognize the limitations of this process. Future research could identify real queries linked to suicide-seeking behavior from non-English speaking populations.

We ran the queries on Google, Bing, and DuckDuckGo. Google and Bing are the most popular search engines in the US. We chose DuckDuckGo because it is known for its stricter privacy protections, which could be attractive to someone thinking of hurting themselves.

We collected the URLs for the first 20 queries for each platform. ${ }^{2}$ We also collected data on whether the search engine itself shows any help messaging (we will refer to these as "direct answer support information"), URLs for the first three advertisements shown, and the top six recommended searches. See Figures 1 and 2 on the next page for examples of the data we collected.

We used the Chrome browser given its high market share (StatCounter, n.d.). We chose to focus only on Chrome after running all Google search queries in English on Chrome, Firefox, and Safari and observing that the results were nearly identical. We similarly decided to focus on desktop searching after piloting queries on mobile devices and observing that the results did not meaningfully vary from desktop searches. All searches were run in incognito mode.

This data collection effort resulted in a primary dataset of 1,200 search result URLs (840 unique URLs). All URLs were double-coded by members of the research team and one outside research assistant. URLs from Spanish queries were coded by a native Spanish speaker and two individuals with high professional-level proficiency in Spanish. Discrepancies were reviewed; if either coder indicated that the URL contained problematic content, it was coded as such. Each URL was coded on 24 attributes related to content (e.g., facts about suicide, news about a suicide death) and type (e.g., a government report, a discussion board), shown in Table 2 on page 7. In analysis, we considered the following attributes to be harmful:

- Encouraging, promoting, or facilitating suicide (for example, a discussion forum where people share recommendations about how to kill oneself by cutting one's wrist).

- Discuss suicide options (for example, a forum where people share thoughts on the least painful way to kill oneself; see also Figure 3 on the next page).

We separately assessed whether the URL itself (as opposed to the search result page) contains a help message. We refer to these as "URL support information."

We were intentionally conservative in what we defined as problematic. For example, we did not code a site that described the lethal overdose amount for Tylenol in the context of providing people with information to stay safe as "discuss suicide options," though the site could provide someone with information on how to self-harm.

2. We chose the number 20 because we wanted to ensure we captured all results on the first page of results, and Bing sometimes links out to video results between its more standard search results. 


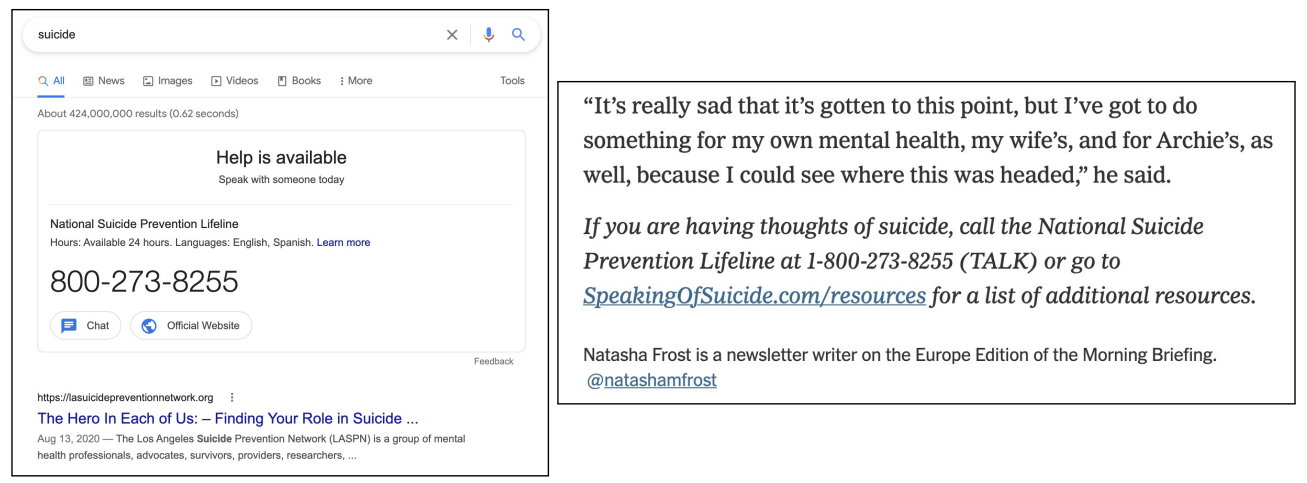

Figure 1: Left, direct answer support information on Google. Right, URL support information at the bottom of a New York Times article.

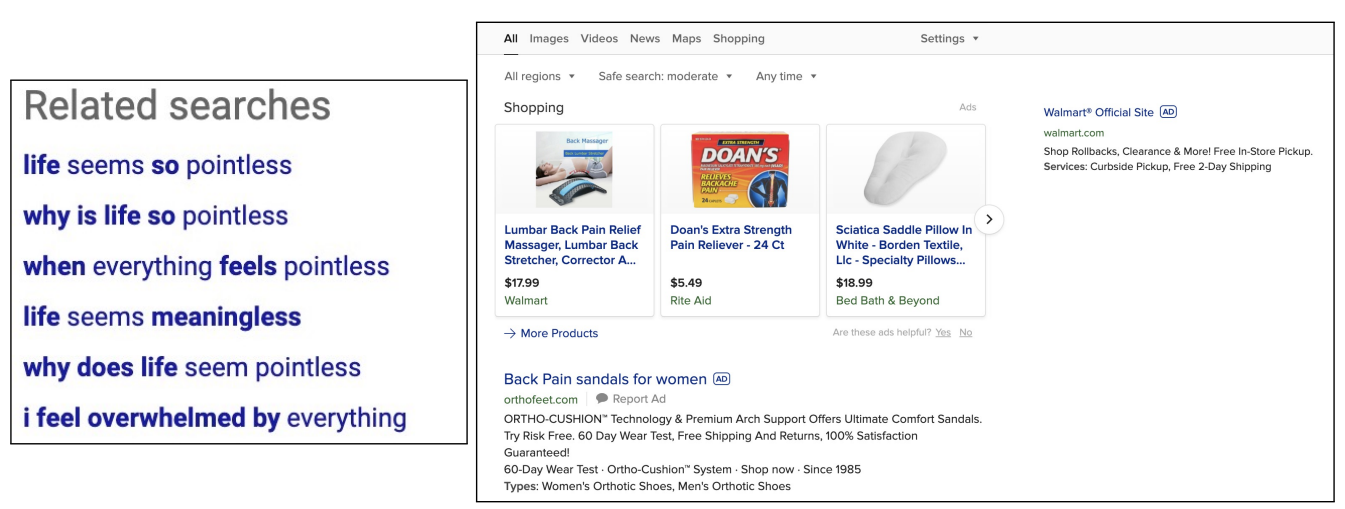

Figure 2: Left, recommended searches on Bing. Right, five advertisements on DuckDuckGo.

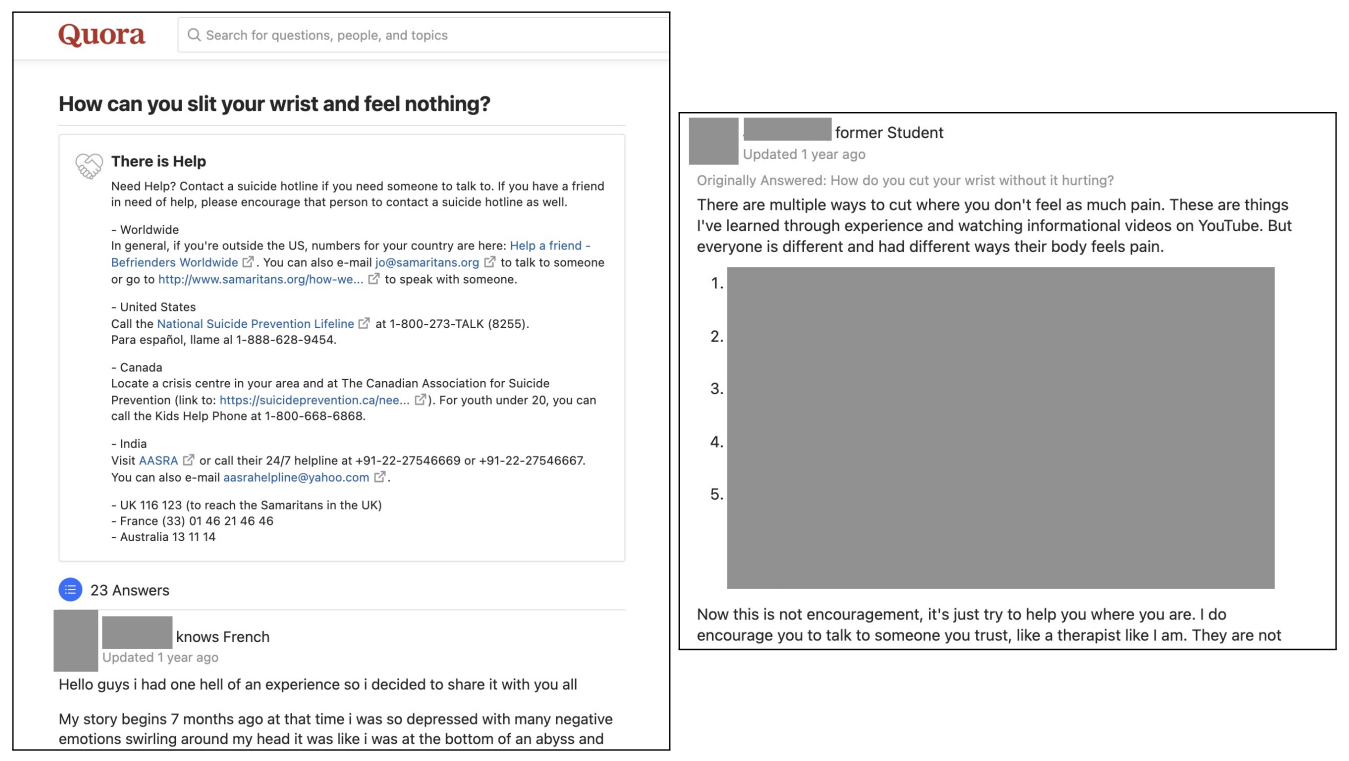

Figure 3: An example URL that we coded as "discuss suicide options." This result was surfaced from a Bing query for "how painful is slitting your wrists." Both screenshots are from the same URL. 
The category "irrelevant" included this example-information about overdosing that was not in the context of suicide-along with definitions of words that could be related to suicide (e.g., a URL providing the definition of affixiation). We also coded URLs about mental health issues that were unrelated to suicide as "irrelevant."

\begin{tabular}{|l|l|}
\hline Content variables (check all that apply) & Type variables (check all that apply) \\
\hline $\begin{array}{l}\text { Encouraging, promoting, or facilitating } \\
\text { suicide }\end{array}$ & Chat room \\
\hline Discuss suicide options & Social media \\
\hline Facts about suicide & Blog \\
\hline $\begin{array}{l}\text { Personal or other accounts of suicide or } \\
\text { request for help }\end{array}$ & NGO website \\
\hline Jokes about suicide & News site \\
\hline Suicide prevention/support & Video \\
\hline News about a specific suicide & Academic \\
\hline News about suicide in general & Government \\
\hline Academic article & “Medical dot com" \\
\hline Mental health support & Wiki \\
\hline Help message & Other \\
\hline Irrelevant & \\
\hline Other & \\
\hline
\end{tabular}

Table 2: Coded URL attributes. The "Medical dot com" category indicates a seemingly medical site with .com, such as psychologytoday.com.

\section{Findings}

In this section we discuss prevalence of harmful content across platforms by query language and query specificity, and look at what types of URLs have the most harmful content. We assess advertisements and recommended searches, and also look the prevalence of direct answer support information by platform, query language, and query specificity.

\subsection{Prevalence of harmful content across platforms}

Bing and DuckDuckGo had more harmful URLs than Google: 22\% of Bing URLs and 19\% of DuckDuckGo URLs were harmful, compared to $7 \%$ of Google URLs. When looking just at the most harmful content (i.e., URLs that we coded as encouraging suicide), the same pattern was observed: $10 \%$ of Bing URLs encouraged suicide, compared to $8 \%$ for DuckDuckGo and 4\% for Google.

A similar pattern was observed when looking at the portion of relevant URLs that display support information on their website (as opposed to on the search engine itself, which is discussed later), although all sites performed poorly on this metric: $75 \%$ of relevant Bing URLs lack support information, compared to $71 \%$ of DuckDuckGo URLs and $64 \%$ of Google URLs. 


\subsection{Harmful results by query language}

For Bing and DuckDuckGo, the percent of harmful search results for Spanish language queries was $18 \%$ and $15 \%$ lower, respectively, compared to English language queries. Figure 4 and Table 3, Models 5-8 in Appendix A show that these differences are statistically significant. For Google there was no meaningful difference in the percent of harmful search results across Spanish and English queries.

While Spanish queries generally surfaced fewer harmful results, relevant results from Spanish queries were about $18 \%$ more likely to lack support information in the URLS compared to relevant English language results (see Figure 4, and Table 4 on page 18). This pattern, which is statistically significant, holds for all search engines.
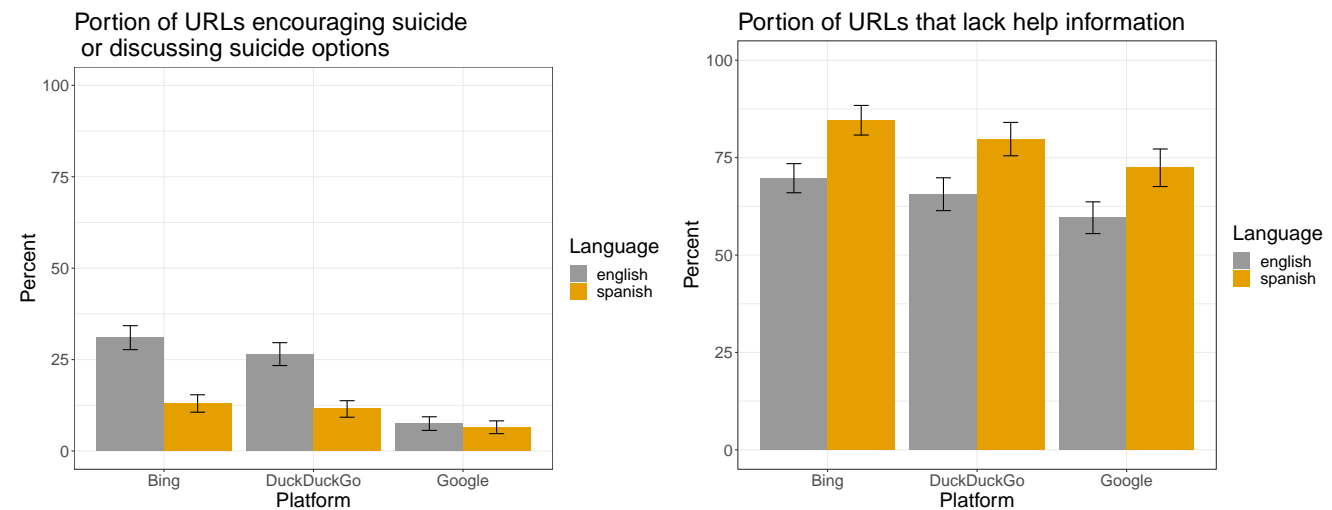

Figure 4: Left, portion of URLs encouraging suicide or discussing suicide options, by platform and language of query. The highest portion of harmful URLs were on Bing and DuckDuckGo in response to English queries. Right, portion of URLs that lack support information by platform and language of query. Relevant Spanish URLs were less likely to contain support information compared to English URLs. For the figure on the right, we exclude URLs that are irrelevant, as it is not reasonable to expect an irrelevant URL to have support information.

\subsection{Harmful results by query specificity}

We find that queries that are more specific are more likely to surface URLs that encourage suicide or discuss suicide options on Bing and DuckDuckGo, but not on Google. Indeed, on Bing, $31 \%$ of search results from specific queries were harmful. Table 3 , Models 6 and 8 , in the Appendix, show that specific queries were $20 \%$ more likely to surface harmful results compared to general queries, and the difference is statistically significant. Specific queries were more likely to surface URLs that lacked help information on Google and DuckDuckGo, but not on Bing (see Table 4 in Appendix A).

\subsection{What types of URLs have the most harmful content?}

Of the $16 \%(\mathrm{~N}=192)$ of the URLs across platforms that encouraged suicide or discussed suicide options, we looked at what types of URLs were most represented. Forty-five percent of these harmful URLs were classified as blogs, and $40 \%$ were classified as social media. Of the harmful URLs in both in English and Spanish, 26 were Quora posts; 22 of the URLs, again across both languages, were from Yahoo Answers, which has since shut down (Statt and Peters 2021). These URLs contained frank discussion of suicide methods and options, many with instructional content or thoughts about the "best" or 

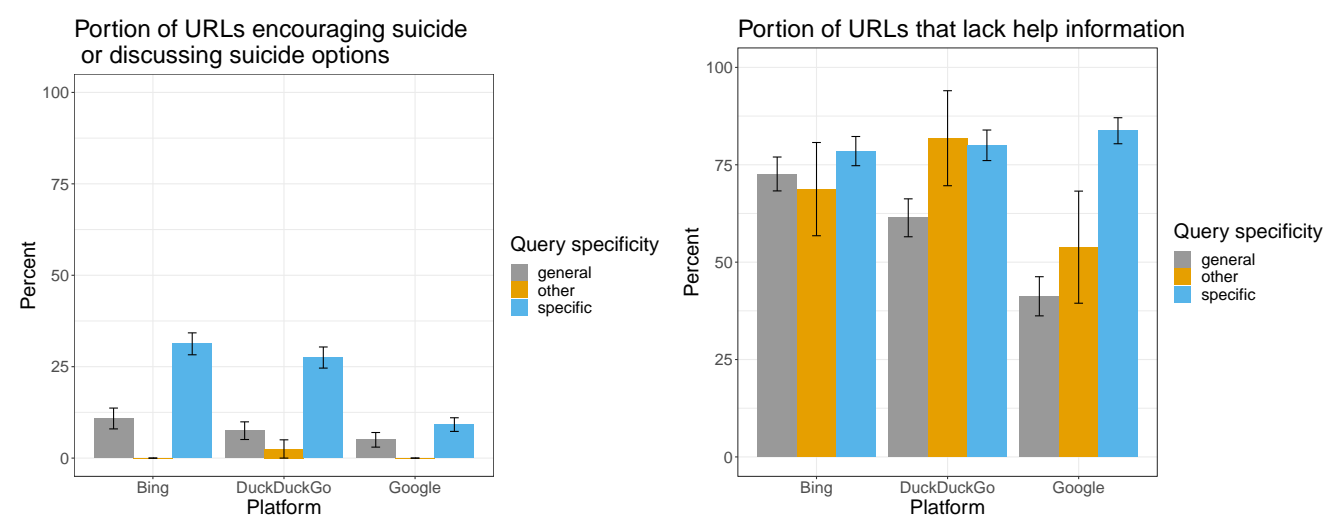

Figure 5: Left, portion of URLs encouraging suicide or discussing suicide options, by platform and query specificity. Specific queries were more likely to surface harmful URLS on Bing and DuckDuckGo, compared to general queries. Right, portion of URLs that lack support information by platform and query specificity. Specific queries were more likely to surface URLs that lacked help information on Google and DuckDuckGo, compared to general queries. For the figure on the right, we exclude URLs that are irrelevant, as it is not reasonable to expect an irrelevant URL to have support information.

least painful way to commit suicide. Several posts began with a disclaimer that the author was not attempting to promote suicide, but in fact the author did just that. Interestingly, $73 \%$ of the Quora URLs contained support information, compared to just $17 \%$ of URLs in the dataset overall. ${ }^{3}$

\subsection{Few and unproblematic advertisements}

We decided to collect data on the first three advertisements shown in each search result. For common queries on Bing (like "car"), more than three advertisements are shown, but our preliminary investigations suggested suicide queries were surfacing far fewer advertisements. Assuming this possibility of three advertisement slots per query, we found that just $6 \%$ of these slots were filled. The dataset included only 10 advertisements (five from DuckDuckGo, three from Google, and two from Bing). Three ads were from English queries and seven from Spanish queries. We investigated these 10 advertisements: none encouraged suicide nor discussed suicide options. The advertisements were either irrelevant (e.g., an advertisement for a back pain device from Target) or discouraged self-harm (e.g., a religious site explaining that there is hope).

\subsection{Prevalence of direct answer support information by platform, query language, and query specificity}

Fifty-five percent of Google queries included direct answer support information, compared to 35\% for Bing and 10\% for DuckDuckGo. English queries were more likely to surface direct answer support information: $53 \%$ of English queries had this information, compared to $13 \%$ of Spanish queries, a statistically significant difference (see Table 5 on page 19). The queries that were most likely to surface direct answer support information were "suicide" and "kill yourself," in English. The query that was least likely to provide a direct answer support information (it never surfaced direct answer support information across platform and language) was "everything seems overwhelming and pointless."

3. However, only one of the four Spanish language Quora URLs contained support messaging. 
This was the only query in the study that did not explicitly mention suicide or a suicide method. More generally, specific queries were $36 \%$ less likely to return direct answer support information compared to general queries.
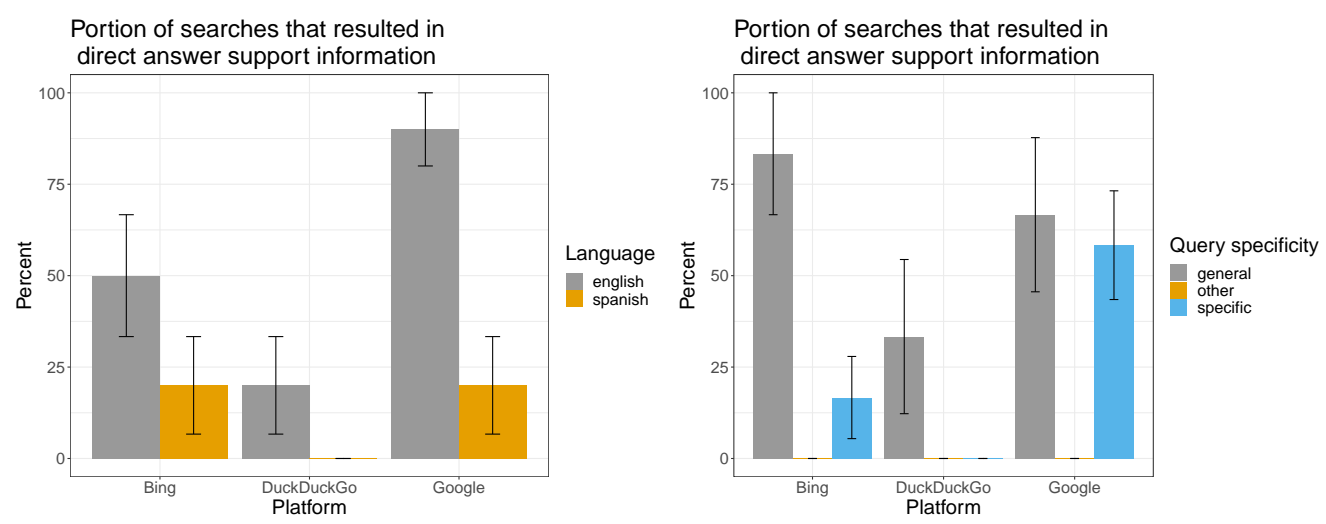

Figure 6: Left, portion of searches that surfaced direct answer support information on the search platform, by language and platform. Google queries returned the most direct answer support information. Right, portion of searches that surfaced direct answer support information on the search platform, by query specificity and platform. Specific queries were less likely than general queries to return direct answer support information.

\subsection{Recommended searches}

We collected data on six recommended searches per query. We chose this number because our preliminary investigation suggested this was the maximum number of recommended searches for suicide-related queries.

Assuming the possibility of six recommended searches per query, for English queries, $19 \%$ of these slots were filled. For these English queries, $100 \%$ of the filled slots were found on Bing. DuckDuckGo did not show any recommended searches across queries, both in English and Spanish. On Bing's recommended searches, $44 \%$ could be conceived as redirecting users to content encouraging self-harm or harm to animals, while $56 \%$ of recommended queries would likely direct users to support resources. Examples of the problematic recommended searches include "easy pills to overdose with," "how long to suffocate self," and "best way to poison dog." Examples of positive search recommendations include "suicide hotline" and "help for depression." The queries with supportive search recommendations were general: "suicide chatrooms," "suicide," and "kill yourself." The queries with problematic search recommendations were specific: "what is the easiest way to poison myself?" "easiest way to suffocate yourself," "everything seems overwhelming and pointless," and "best pills to overdose on."

Assuming the possibility of six recommended searches per query, for Spanish queries, $17 \%$ of these slots were filled. Of these filled slots, $87 \%$ were found on Google and $13 \%$ were found on Bing. Eleven percent of Google's recommended searches were harmful, including "cual es el modo más fácil de envenenarse" "“what is the easiest way to poison yourself"). None of Bing's recommended searches were harmful. 


\section{Discussion}

The goal of this study was to describe the results returned across three search engines for suicide-specific and suicide means-related searches in English and Spanish. We found that Bing and DuckDuckGo return more harmful URLs results when compared with Google. They were also less likely to display help messaging at the top of search results. Specific queries related to suicide means were more likely to generate harmful results compared with more general queries with obvious suicide-related terms. Although we anticipated more problematic (pro-suicide results) in Spanish searches, we found that Spanish queries resulted in considerably fewer harmful results than English queries. However, Spanish searches produced fewer prevention-focused direct answer support information. None of the platforms, across queries and languages, showed a single harmful advertisement. However, there were a number of harmful recommended searches. These harmful recommended searches were most common on Bing and in English.

When we further investigated harmful URLs, we found that many (both in English and Spanish) were from Quora (though a majority of these had help messaging within the Quora page) and the now-defunct Yahoo Answers. Although there were fewer harmful results produced for Spanish language searches, this may be due to the relative lack of Spanish content on the internet, rather than greater effectiveness at filtering out harmful results. The Spanish-language Quora site is relatively new (five years), and likely has fewer posts that would fit the search terms. It is also possible that Spanish speakers are less likely to post pro-suicide content; more than $50 \%$ of Spanish speakers in the United States identify as Catholic, and another $30 \%$ identify as other Christian denominations. Religious beliefs are known to be protective against suicide, and Catholicism teaches that suicide is a sin against God.

Given the established risk of suicide contagion, the low prevalence of problematic results on Google compared with Bing and DuckDuckGo is encouraging. Given Google's relative market share, this may reflect an internal commitment to dedicate resources to preemptively audit problematic posts. Research suggests that individuals who attempt and complete suicide expend considerable efforts to hide their preparatory plans from loved ones and others with whom they interact. They may thus use lesser-known search engines such as DuckDuckGo because of their relative privacy. Search engines with more modest market shares should consider enhancing efforts to audit harmful results in response to queries about suicide.

Of note, means-related searches were more likely to return problematic results. Given that these searches were pulled from actual searches by individuals contemplating suicide (i.e., more representative of the types of queries people make), there is significant room for improvement in algorithms designed to detect suicide content. People making specific searches may be at higher risk to attempt or complete suicide, and failure to proactively audit specific queries may have dire consequences.

We were encouraged to see no harmful advertisements across platforms in our dataset. This may be because the platforms actively monitor advertisements for self-harm queries; a more likely explanation is that advertisers are unlikely to include terms related to suicide among the ad words they select.

The negligible inclusion of suicide prevention hotline information on Bing and DuckDuckGo highlights gaps between search platforms' purported goals and their actual performance in suicide prevention. 
Our results are consistent with those from previous work suggesting that search engines likely rely on simple keyword algorithms to determine whether to display hotline information. More sophisticated methods may more effectively target a broad range of suicide-related searches (Arendt, Haim, and Scherr 2020).

\section{Conclusion}

Preventing suicidal and other self-harm behaviors is an urgent public health imperative. Suicide rates have consistently climbed over the past decade. Further, there is global concern that the mental health sequelae of the COVID-19 pandemic may exacerbate risk for suicidal ideation, self-harm, and completed suicide (Mayer et al. 2020). The technology sector overseeing prominent search engines is uniquely positioned to scrutinize suicide-related content with the basic goal of reducing human suffering. However, to effectively reduce harmful search results while also promoting suicide prevention hotlines and mental health resources, these platforms must invest more to better understand the types of searches that individuals considering suicide make. Focusing only on face-valid terms neglects a majority of searches related to actual means, which are more likely to result in harm. 


\section{References}

Arendt, Florian, Mario Haim, and Sebastian Scherr. 2020. "Investigating Google's suicideprevention efforts in celebrity suicides using agent-based testing: a cross-national study in four European countries." Social Science \& Medicine 262:112692.

Arendt, Florian, and Sebastian Scherr. 2017. "Optimizing online suicide prevention: A search engine-based tailored approach.” Health Communication 32 (11): 14031408.

Arendt, Florian, Sebastian Scherr, and Daniel Romer. 2019. "Effects of exposure to selfharm on social media: Evidence from a two-wave panel study among young adults." New Media \& Society 21 (11-12): 2422-2442.

Ayers, John W, Benjamin M Althouse, Eric C Leas, Mark Dredze, and Jon-Patrick Allem. 2017. "Internet searches for suicide following the release of 13 Reasons Why." JAMA Internal Medicine 177 (10): 1527-1529.

Becker, Katja, and Martin H Schmidt. 2004. "Internet chat rooms and suicide." Journal of the American Academy of Child \& Adolescent Psychiatry 43 (3).

Birnbaum, Michael L, Asra F Rizvi, Christoph U Correll, John M Kane, and Jamie Confino. 2017. "Role of social media and the Internet in pathways to care for adolescents and young adults with psychotic disorders and non-psychotic mood disorders." Early Intervention in Psychiatry 11 (4): 290-295.

Bridge, Jeffrey A, Joel B Greenhouse, Donna Ruch, Jack Stevens, John Ackerman, Arielle H Sheftall, Lisa M Horowitz, Kelly J Kelleher, and John V Campo. 2020. "Association between the release of Netflix's 13 Reasons Why and suicide rates in the United States: An interrupted time series analysis." Journal of the American Academy of Child \& Adolescent Psychiatry 59 (2): 236-243.

Carlyle, Kellie E, Jeanine PD Guidry, Kofoworola Williams, Ariella Tabaac, and Paul B Perrin. 2018. "Suicide conversations on Instagram ${ }^{\text {TM }}$ : contagion or caring?" Journal of Communication in Healthcare 11 (1): 12-18.

Christensen, Helen, Philip J Batterham, and Bridianne O'Dea. 2014. “E-health interventions for suicide prevention." International Journal of Environmental Research and Public Health 11 (8): 8193-8212.

Constine, Josh. 2019. "Microsoft Bing not only shows child sexual abuse, it suggests it," https://techcrunch.com/2019/01/10/unsafe-search/.

Daine, Kate, Keith Hawton, Vinod Singaravelu, Anne Stewart, Sue Simkin, and Paul Montgomery. 2013. "The power of the web: a systematic review of studies of the influence of the internet on self-harm and suicide in young people." PloS One 8 (10): e77555.

Dunlop, Sally M, Eian More, and Daniel Romer. 2011. "Where do youth learn about suicides on the Internet, and what influence does this have on suicidal ideation?" Journal of Child Psychology and Psychiatry 52 (10): 1073-1080.

Eggertson, Laura. 2015. Social media embraces suicide prevention, 11.

Facebook. 2021. "Suicide and Self-Injury" (September 30, 2021). Accessed October 27, 2021. https://transparency.fb.com/policies/community-standards/suicide-selfinjury/.

Joiner Jr, Thomas E. 1999. “The clustering and contagion of suicide." Current Directions in Psychological Science 8 (3): 89-92. 
Keller, Michael H., and Gabriel J.X. Dance. 2019. "Child Abusers Run Rampant as Tech Companies Look the Other Way." New York Times, https:// www. nytimes.com/ interactive/2019/11/09/us/internet-child-sex-abuse.html.

Khasawneh, Amro, Kapil Chalil Madathil, Emma Dixon, Pamela Wiśniewski, Heidi Zinzow, Rebecca Roth, et al. 2020. "Examining the self-harm and suicide contagion effects of the Blue Whale Challenge on YouTube and Twitter: qualitative study." JMIR Mental Health 7 (6): e15973.

Kirtley, Olivia J, and Rory C O'Connor. 2020. "Suicide prevention is everyone's business: challenges and opportunities for Google." Social Science \& Medicine 262:112691.

Klimes-Dougan, Bonnie, Nathan Wright, and David A Klingbeil. 2016. "Suicide prevention public service announcements impact help-seeking attitudes: The message makes a difference." Frontiers in Psychiatry 7:124.

Lai, Mee Huong, Thambu Maniam, Lai Fong Chan, and Arun V Ravindran. 2014. "Caught in the web: a review of web-based suicide prevention." Journal of Medical Internet Research 16 (1): e2973.

Luby, Joan, and Sarah Kertz. 2019. "Increasing suicide rates in early adolescent girls in the United States and the equalization of sex disparity in suicide: the need to investigate the role of social media." JAMA Network Open 2 (5): e193916-e193916.

Marchant, Amanda, Keith Hawton, Ann Stewart, Paul Montgomery, Vinod Singaravelu, Keith Lloyd, Nicola Purdy, Kate Daine, and Ann John. 2017. "A systematic review of the relationship between internet use, self-harm and suicidal behaviour in young people: The good, the bad and the unknown." PLoS One 12 (8): e0181722.

Mars, Becky, Jon Heron, Lucy Biddle, Jenny L Donovan, Rachel Holley, Martyn Piper, John Potokar, Clare Wyllie, and David Gunnell. 2015. "Exposure to, and searching for, information about suicide and self-harm on the Internet: Prevalence and predictors in a population based cohort of young adults." Journal of Affective Disorders 185:239245.

Massing-Schaffer, Maya, and Jacqueline Nesi. 2020. "Cybervictimization and suicide risk in adolescence: An integrative model of social media and suicide theories." Adolescent Research Review 5 (1): 49-65.

Mayer, Lea, Nicolas Rüsch, Laura M Frey, Michael R Nadorff, Chris W Drapeau, Lindsay Sheehan, and Nathalie Oexle. 2020. "Anticipated suicide stigma, secrecy, and suicidality among suicide attempt survivors." Suicide and Life-Threatening Behavior 50 (3): 706-713.

Moon, Khatiya C., Anna Van Meter, Michael A. Kirschenbaum, Asra Alil, John M. Kanel, and Michael L. Birnbaum. in press. "Internet search activity of young people with mood disorders hospitalized for suicidal thoughts and behaviors: a qualitative study of Google search activity." In press.

Niederkrotenthaler, Thomas, Steven Stack, Benedikt Till, Mark Sinyor, Jane Pirkis, David Garcia, Ian RH Rockett, and Ulrich S Tran. 2019. "Association of increased youth suicides in the United States with the release of 13 Reasons Why." JAMA Psychiatry 76 (9): 933-940.

Perkins, Shelby, Elena Cryst, and Shelby Grossman. 2021. "Sizing Up Self-Harm Policies." Stanford Internet Observatory, https://fsi.stanford.edu/self-harm-policies-report. 
Randall, Jason R, Nathan C Nickel, and Ian Colman. 2015. "Contagion from peer suicidal behavior in a representative sample of American adolescents." Journal of Affective Disorders 186:219-225.

Reporting on Suicide. n.d. Best Practices and Recommendations for Reporting on Suicide. Accessed October 27, 2021. https://reportingonsuicide.org/wp-content/themes/ ros2015/assets/images/ROS-001-One-Pager-1.13.pdf.

"Multilingual SEO: It's Actually a Pretty Big Challenge for Google to Determine the Language of a Query." 2014. Search Engine Journal, https://www.searchenginejournal. $\mathrm{com} /$ multilingual-seo-actually-pretty-big-challenge-google-determine-languagequery/107871/.

Sedgwick, Rosemary, Sophie Epstein, Rina Dutta, and Dennis Ougrin. 2019. "Social media, internet use and suicide attempts in adolescents." Current Opinion in Psychiatry 32 (6): 534.

Sinyor, Mark, Marissa Williams, Ulrich S Tran, Ayal Schaffer, Paul Kurdyak, Jane Pirkis, and Thomas Niederkrotenthaler. 2019. "Suicides in young people in Ontario following the release of "13 Reasons Why"." The Canadian Journal of Psychiatry 64 (11): 798-804.

Sisask, Merike, and Airi Värnik. 2012. "Media roles in suicide prevention: a systematic review." International Journal of Environmental Research and Public Health 9 (1): 123-138.

“Bing's Top Search Results Contain an Alarming Amount of Disinformation.” 2019. Stanford Internet Observatory, https://cyber.fsi.stanford.edu/io/news/bing-searchdisinformation.

StatCounter. n.d. Browser Market Share Worldwide. Accessed October 27, 2021. https: //gs.statcounter.com/browser-market-share.

Statt, Nick, and Jay Peters. 2021. "Yahoo Answers will be shut down forever on May 4th,” https://www.theverge.com/2021/4/5/22368488/yahoo-answers-shutdownmay-4-internet-era-over-rip.

Sugg, Margaret M, Kurt D Michael, Scott E Stevens, Robert Filbin, Jaclyn Weiser, and Jennifer D Runkle. 2019. "Crisis text patterns in youth following the release of 13 Reasons Why Season 2 and celebrity suicides: A case study of summer 2018." Preventive Medicine Reports 16:100999.

Sumner, Steven A, Moira Burke, and Farshad Kooti. 2020. "Adherence to suicide reporting guidelines by news shared on a social networking platform." Proceedings of the National Academy of Sciences 117 (28): 16267-16272.

TikTok. n.d. "Suicide \& self-harm," accessed October 27, 2021. https://www.tiktok.com/ safety/en/suicide-self-harm/.

Van Meter, Anna R, Michael L Birnbaum, Asra Rizvi, and John M Kane. 2019. “Online help-seeking prior to diagnosis: Can web-based resources reduce the duration of untreated mood disorders in young people?" Journal of Affective Disorders 252:130134.

Velting, Drew M, and Madelyn S Gould. 1997. “Suicide contagion.” Review of Suicidology.

Won, Hong-Hee, Woojae Myung, Gil-Young Song, Won-Hee Lee, Jong-Won Kim, Bernard J Carroll, and Doh Kwan Kim. 2013. "Predicting national suicide numbers with social media data." PloS One 8 (4): e61809. 


\section{Authors}

Olivia Borge is a Research Assistant with Victoria Cosgrove at Stanford University.

Victoria Cosgrove is a Clinical Associate Professor in the Division of Child and Adolescent Psychiatry at the Stanford University School of Medicine.

Elena Cryst is the Assistant Director of the Stanford Internet Observatory.

Shelby Grossman is a Research Scholar at the Stanford Internet Observatory.

Shelby Perkins is a recent graduate of Stanford University's Masters in International Policy Program.

Anna Van Meter is an Assistant Professor at the Institute of Behavioral Science, Feinstein Institutes for Medical Research, and an Assistant Professor at the Donald and Barbara Zucker School of Medicine at Hofstra/Northwell.

\section{Acknowledgements}

We thank Maria Fernanda Porras Jacobo for research assistance and two anonymous reviewers for helpful feedback. Shelby Perkins is currently employed by Twitter, but all of her contributions to this paper occurred while she was a Research Assistant with the Stanford Internet Observatory.

\section{Funding Statement}

Not applicable.

\section{Ethical Standards}

To avoid the possibility of pressuring student research assistants into viewing pro-suicide content, the authors of this paper were the primary coders of the URL dataset. We brought in one research assistant due to the need for one additional coder who spoke Spanish. Students involved in this project were encouraged to use filters that made their screens black and white to limit the effect of exposure to harmful content. Our coding protocol also prohibited spending more than 30 seconds viewing a URL.

\section{Keywords}

Search engines; suicide; self harm 


\section{Appendices}

\section{Appendix A: Harmful content, platforms, languages, and query specificity}

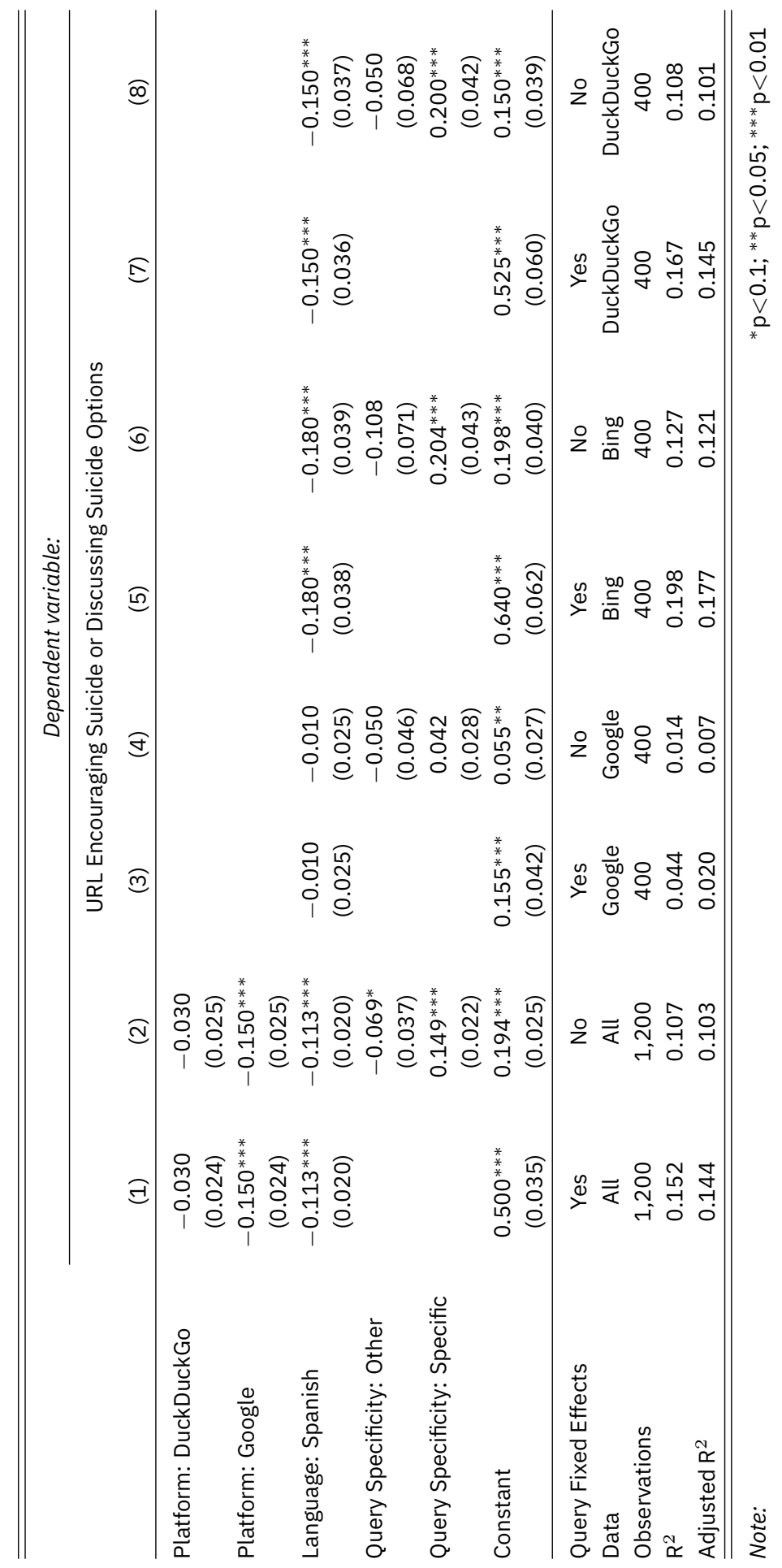

Table 3: Reference categories include: Platform: Bing, Language: English, and Query Specificity: General. 


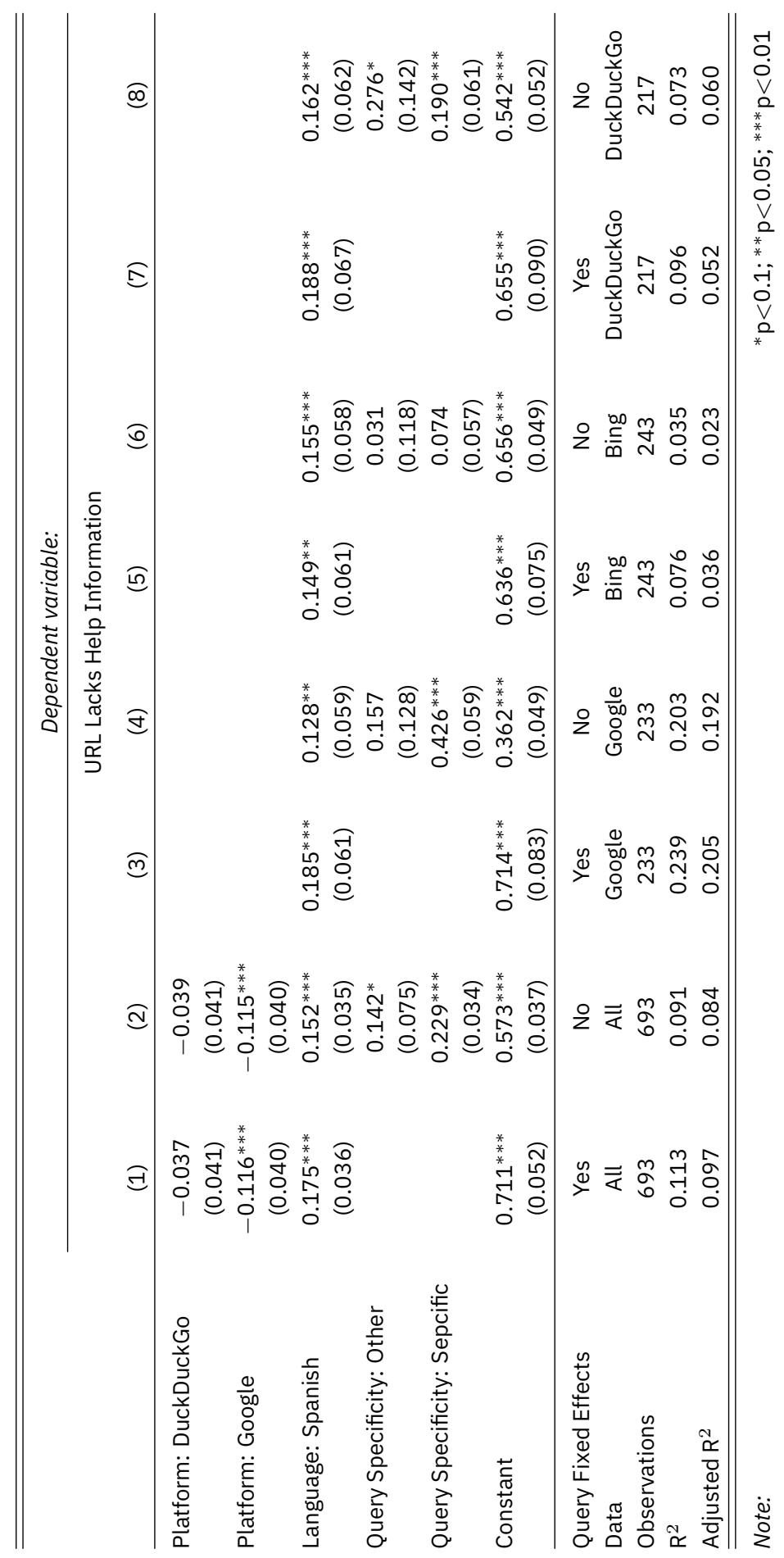

Table 4: Sample size varies across models as these models exclude irrelevant URLs. Reference categories include: Platform: Bing, Language: English, and Query Specificity: General. 


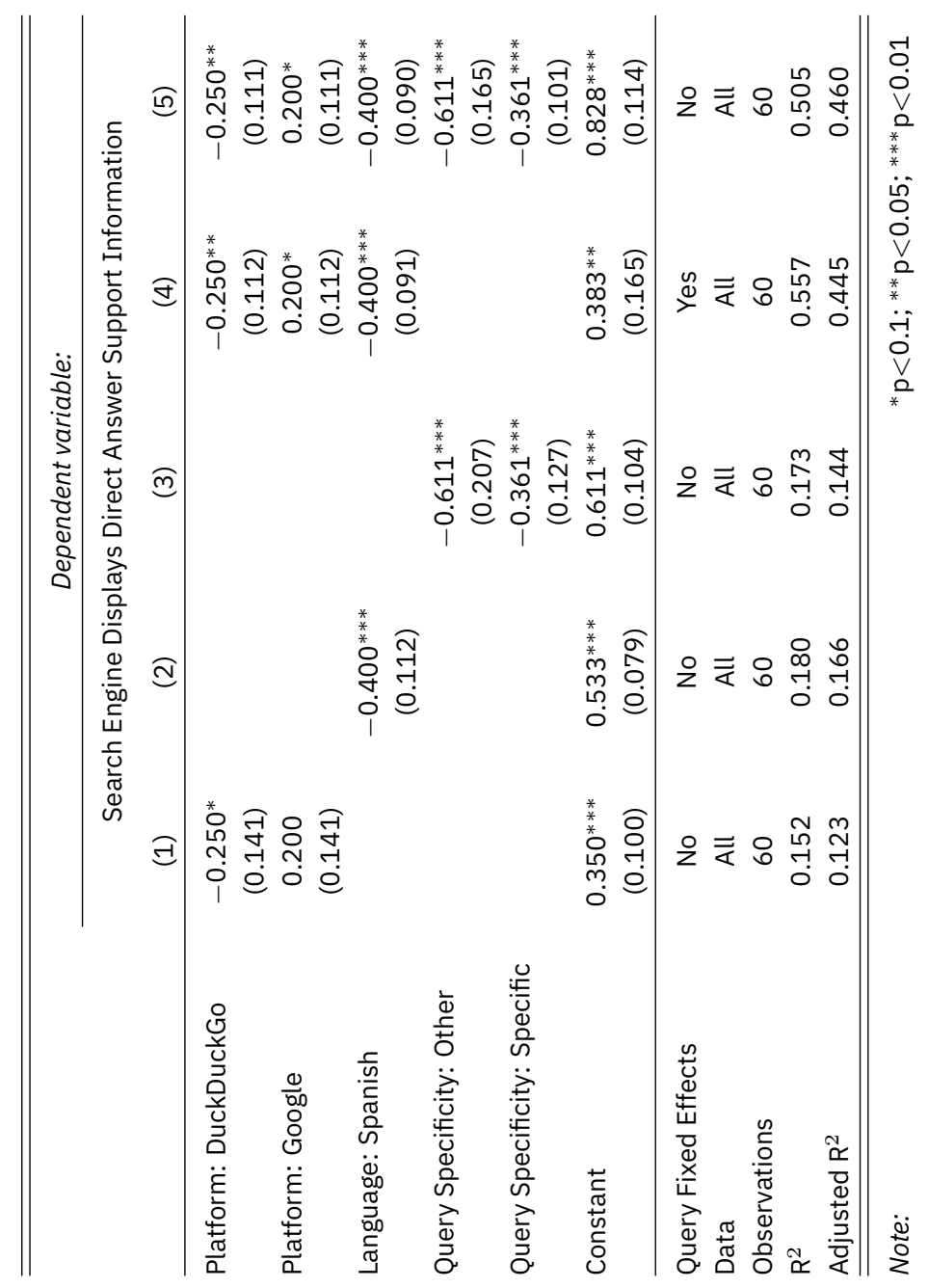

Table 5: Reference categories include: Platform: Bing, Language: English, and Query Specificity: General. 